\title{
Do no harm - but first, do not hurt
}

\section{Raymond D. Pitetti}

ß See related article page 1691

A $s$ recently as 20 years ago, many health care professionals believed that young children did not experience pain and that the use of opiates for pain control was contraindicated because of a substantial risk of addiction. ${ }^{1} \mathrm{~A}$ related misunderstanding was the belief that even if children experienced pain, they would not remember it, and therefore they would sustain no lasting effects. Another commonly held belief was that a child's pain could not be measured accurately. As a result, many clinicians performed painful procedures, including intravenous cannulation, on children without regard for the pain the child was experiencing.

Thankfully, we no longer hold to such archaic ideas. Neuroanatomic studies have shown that by 29 weeks of gestation, pain pathways and the cortical and subcortical centres involved in the perception of pain are well developed, as are the neurologic systems for transmitting and modulating painful sensations. Therefore, even fetuses can perceive pain in ways similar to those of an older child. ${ }^{2}$ In addition, studies have shown that pain and distress, such as that associated with painful procedures, can endure in the memory and result in, for example, disturbances to eating, sleeping and the stability of the state of arousal. ${ }^{3}$ Finally, pain in children can be assessed through physiologic indicators, systematic observation of behaviour, and reports by the children themselves. As a result, there has been a marked change in the approach to pain and pain management in children.

Some practitioners continue to believe that it is easier and safer not to provide anesthesia or analgesia to children before painful procedures. Belying this belief are the results of numerous studies showing that infants and children can receive anesthesia and analgesia safely with proper age-related adjustments in clinical practice and dosing. ${ }^{4}$ Moreover, new topical anesthetics now allow for pain-free delivery of local anesthetics. Thus, it is no longer acceptable not to consider the pain and stress associated with a painful procedure.

Taddio and colleagues have conducted a double-blind, randomized controlled trial comparing liposomal lidocaine $4 \%$ cream (Maxilene) with placebo during intravenous cannulation among children (see page 1691). They were primarily concerned with successful intravenous insertion and the amount of pain the child experienced during the procedure. As they relate, this formulation of lidocaine has a shorter application time than lidocaine-prilocaine $5 \%$ cream and is less likely than amethocaine $4 \%$ gel to cause changes to the skin (vasodilatation) that would make cannulation difficult. Liposomal lidocaine has been found to compare favourably with other local anesthetics with respect to analgesia. However, because it had never been compared with placebo, its impact on procedure success rates was unknown.
As Taddio and colleagues report, use of liposomal lidocaine before intravenous cannulation resulted in significantly higher success rates and a shorter overall procedure time compared with placebo. As well, children experienced substantially less pain during the procedure. The authors conclude that the increase in success rates was most likely the result of pain relief and the minimal skin changes associated with the cream.

Liposomal lidocaine has the advantages of a short application time, needle-free administration and minimal vasoactive properties. Thus, it is ideal for procedures such as intravenous cannulation. Many clinicians believe that topical anesthetics will interfere with the successful completion of the procedure. However, Taddio and colleagues have shown that this does not occur with liposomal lidocaine.

As clinicians caring for children, we should be cognizant of the consequences of our actions. It is clear that children experience pain. Thus, we should make every effort to alleviate or mitigate this pain, especially during painful procedures. Taddio and colleagues have shown that the use of a topical anesthetic such as liposomal lidocaine can result in significantly less pain perceived by the child and a higher procedure success rate. In fact, successful anesthesia and analgesia may in some cases obviate the need for procedural sedation. Clinicians have an ethical responsibility to provide full treatment of pain in children unless otherwise justified by defined therapeutic benefits. The assessment and treatment of pain in children are important aspects of pediatric care, and failure to provide adequate control of pain amounts to substandard and unethical medical practice. Taddio and colleagues have provided us with one more therapeutic option.

This article has been peer reviewed.

Dr. Pitetti is an Assistant Professor of Pediatrics in the Division of Pediatric Emergency Medicine at the Children's Hospital of Pittsburgh, Pittsburgh, Penn.

Competing interests: None declared.

\section{References}

1. Wagner AM. Pain control in the pediatric patient. Dermatol Clin 1998;16:609-17.

2. Mathew PJ, Mathew JL. Assessment and management of pain in infants. Postgrad Med f 2003;79:438-43.

3. Marshall RE, Stratton WC, Moore JA, Boxerman SB. Circumcision: I. Effects upon newborn behavior. Infant Behav Dev 1980;3:1-14.

4. Bedre CB, Sethna NF. Drug therapy: analgesics for the treatment of pain in children. N Engl f Med 2002;337:1094-103.

5. Taddio A, Soin HK, Schuh S, Koren G, Scolnik D. Liposomal lidocaine to improve procedural success rates and reduce procedural pain among children: a randomized controlled trial. CMA7 2005:172(13):1691-5.

Correspondence to: Dr. Raymond D. Pitetti, Division of Pediatric Emergency Medicine, Children's Hospital of Pittsburgh, 3705 Fifth Ave., Pittsburgh PA 15213; fax 412 692-7464;

raymond.pitetti@chp.edu 